\title{
Large-eddy simulation of circular cylinder flow at subcritical Reynolds number: Turbulent wake and sound radiation
}

\author{
Li Guo ${ }^{1,2}$ - Xing Zhang ${ }^{2}$ - Guowei He ${ }^{2}$
}

Received: 15 May 2015 / Revised: 21 August 2015 / Accepted: 21 September 2015 / Published online: 31 October 2015

(C) The Chinese Society of Theoretical and Applied Mechanics; Institute of Mechanics, Chinese Academy of Sciences and Springer-Verlag Berlin Heidelberg 2015

\begin{abstract}
The flows past a circular cylinder at Reynolds number 3900 are simulated using large-eddy simulation (LES) and the far-field sound is calculated from the LES results. A low dissipation energy-conserving finite volume scheme is used to discretize the incompressible NavierStokes equations. The dynamic global coefficient version of the Vreman's subgrid scale (SGS) model is used to compute the sub-grid stresses. Curle's integral of Lighthill's acoustic analogy is used to extract the sound radiated from the cylinder. The profiles of mean velocity and turbulent fluctuations obtained are consistent with the previous experimental and computational results. The sound radiation at far field exhibits the characteristic of a dipole and directivity. The sound spectra display the $-5 / 3$ power law. It is shown that Vreman's SGS model in company with dynamic procedure is suitable for LES of turbulence generated noise.
\end{abstract}

Keywords Flows past circular cylinder - Aerodynamic noise $\cdot$ Large-eddy simulation $\cdot$ Unstructured grid $\cdot$ Acoustic analogy

$\triangle$ Guowei He

hgw@lnm.imech.ac.cn

1 China Academy of Aerospace Aerodynamics, Beijing 100074, China

2 State Key Laboratory of Non-linear Mechanics, Institute of Mechanics, Chinese Academy of Sciences, Beijing 100190, China

\section{Introduction}

Large-eddy simulation (LES) is a promising approach for numerical predictions of turbulence-generated noise. In LES, large-scale eddies are explicitly resolved while small-scale ones are not resolved, but their effects on large-scale ones are modeled by so-called sub-grid scale (SGS) models. In comparison with the conventional Reynolds averaging Navier-Stokes approach, LES can represent the unsteady properties of larger-scale eddies, which is dominantly important to turbulence-generated noise [1]. On the other hand, LES requires much less computation costs than the direct numerical simulation approach. In the application of LES to turbulence-generated noise, Marsden et al. [2] used LES to calculate trailing edge noise. In addition, Yang et al. [3] used LES to calculate the noise from boundary layer with roughness. Also, Spalart et al. [4] predicted the sound radiating from landing gears using detached eddy simulation. Meanwhile, Boudet et al. [5] and Seo et al. [6] used LES to calculate the sound generated by a cylinder at the Reynolds number $4.6 \times 10^{4}$. Moreover, Orselli et al. [7] investigated a similar case at the Reynolds number $9.0 \times 10^{4}$, and Li et al. [8] studied the mixing layer noise.

Flows past a circular cylinder are the prototype for many industrial applications, especially for the landing gear of airplanes [4]. It raises several great challenges to LES to calculate the flow fields and their radiated noise at the Reynolds number 3900, which is corresponding to the subcritical regime [9]. In this case, the boundary layers around the cylinder are laminar and separate to form the shear layers, which transit to turbulent flows in wakes. These flow properties require very fine meshes and the low-dissipation numerical schemes in LES, since the location of the transition point is very sensitive to numerical dissipation. Large numerical dis- 
sipations could delay the transitions and increase the lengths of laminar shear layers [10]. The noise calculations raise two more challenges: the broadband dipole noise generated by pressure fluctuations on the cylinder surfaces and the broadband quadrupole noise by turbulent wake structures. Both of them are largely influenced by numerical dissipations. For those reasons, we will use the discrete kinetic-energy conservative scheme [11] on unstructured meshes: the conservative finite volume scheme with collocated arrangement of variables.

A SGS model is critically important to the LES of noise radiated by flows around cylinders. For example, Smagorinsky's SGS model [12] has been most widely used in the broad spectrum of turbulent flows. However, in the case of flows past a cylinder at $R e=3900$, the boundary layer is laminar and transition occurs in shear layers. The dissipation of the SGS model is required to be as low as possible in the laminar region. The Smagorinsky SGS model does not meet this criterion. Therefore, we will use the recently developed Vremann's SGS model [13]. The eddy-viscosity coefficient in Vremann's model is determined from the second invariant of the velocity gradient tensor. It is rigorously zero for several laminar velocity profiles including the boundary layer and the transition region. This leads to it having a low SGS dissipation in the laminar regions of the present case. The coefficient in Vremann's model is determined using a dynamic procedure proposed by You et al. [14]. This SGS model also prevents a negative eddy-viscosity. However, Vreman SGS model in company with the dynamic procedure has not been used for turbulence-generated noise. The main objective and new development in this paper are to evaluate the performance of the dynamic Vreman SGS model in terms of sound calculation. The typical case for this purpose is the flow around circular cylinder at subcritical Reynolds number $R e=3900$.

The rest of the paper is arranged as follows: Sect. 2 is devoted to describing the LES method of flows pass a cylinder at $R e=3900$ using Vremann's SGS model in combination with the dynamic global coefficient procedure. The Curle's integral of Lighthill's acoustic analogy is used to calculate the far-filed noise in Sect. 3. The results obtained are presented and discussed in Sect. 4. Finally, the summaries are provided in Sect. 5.

\section{Large-eddy simulation of turbulent flow passing a cylinder}

\subsection{Governing equations}

The governing equations used in calculating far-field noise radiated by flows around a circular cylinder are the filtered Navier-Stokes equations

$$
\begin{aligned}
& \frac{\partial \boldsymbol{u}_{j}}{\partial x_{j}}=0 \\
& \frac{\partial \boldsymbol{u}_{i}}{\partial t}+\frac{\partial \boldsymbol{u}_{i} \boldsymbol{u}_{j}}{\partial x_{j}}=-\frac{\partial \bar{p}}{\partial x_{i}}+\frac{1}{R e} \frac{\partial^{2} \boldsymbol{u}_{i}}{\partial x_{j} \partial x_{j}}-\frac{\partial \bar{\tau}_{i j}}{\partial x_{j}},
\end{aligned}
$$

where $i, j=1,2,3$. Spatial coordinates are denoted as $x_{i}$ or $(x, y, z)$, temporal coordinates denoted as $t$, velocity vector denoted as $\boldsymbol{u}_{i}(u, v, w)$, and pressure denoted as $p$. The over bar " "-" represents space filtering. Reynolds number is denoted as $R e=D U_{\infty} / \nu$, where $D$ is the diameter of the cylinder, $U_{\infty}$ incoming flow velocity and $v$ kinematic viscosity. The SGS stresses are denoted as $\bar{\tau}_{i j}$, which is modeled by the eddy-viscosity SGS model as

$\bar{\tau}_{i j}-\frac{1}{3} \delta_{i j} \bar{\tau}_{k k}=-2 v_{t} \bar{S}_{i j}$,

where $\delta_{i j}$ is the Kronecher symbol. The filtered strain rate tensor is $\bar{S}_{i j}$. The eddy-viscosity coefficient $v_{t}$ is calculated from the global coefficient dynamic Vremen's SGS model [14], which will be introduced below.

\subsection{A global coefficient dynamic Vremen's SGS model}

In the sub-critical flow regime, the boundary layer of the cylinder is laminar [9]. The flow transits to turbulence in the shear layer after the separation point. The numerical dissipation provided by the SGS model should be zero in the region of separation and transition, while it should be appropriately large in the turbulent wakes. Incorrectly large dissipation of a SGS model can enlarge the separation zone artificially in the mean velocity profiles, whereas improper small dissipation may erroneously shorten it. In the work by You and Moin [14], a dynamic procedure was proposed to determine the global coefficient in Vreman's model [13]. In the Vreman SGS model, the eddy-viscosity coefficient $v_{t}$ is calculated by using

$v_{t}=C \sqrt{\frac{\bar{B}_{\beta}}{\bar{\alpha}_{i j} \bar{\alpha}_{i j}}}$,

where

$\bar{B}_{\beta}=\bar{\beta}_{11} \bar{\beta}_{22}+\bar{\beta}_{22} \bar{\beta}_{33}+\bar{\beta}_{33} \bar{\beta}_{11}-\bar{\beta}_{12}^{2}-\bar{\beta}_{23}^{2}-\bar{\beta}_{31}^{2}$,

$\bar{\beta}_{i j}=\sum_{m=1}^{3} \bar{\Delta}_{m}^{2} \bar{\alpha}_{m i} \bar{\alpha}_{m j}$.

Here, $\bar{\alpha}_{i j}=\partial \bar{u}_{j} / \partial x_{i}$ is the derivatives of the filtered velocity, $\bar{\Delta}_{m}$ is the filter width in the spatial directions. For an unstructured grid, $\bar{\Delta}_{m}$ is taken as the cubic root of the cell volume for simplicity. The coefficient $C$ is determined dynamically by using 


$$
\begin{aligned}
C= & -\frac{1}{2 \operatorname{Re}}\left\langle\bar{\alpha}_{i j} \bar{\alpha}_{i j}-\hat{\bar{\alpha}}_{i j} \hat{\bar{\alpha}}_{i j}\right\rangle \\
& \times\left\langle\sqrt{\frac{\bar{B}_{\beta}}{\bar{\alpha}_{i j} \bar{\alpha}_{i j}}} \bar{S}_{i j} \bar{S}_{i j}-\sqrt{\frac{\hat{\bar{B}}_{\beta}}{\hat{\bar{\alpha}}_{i j} \hat{\bar{\alpha}}_{i j}}} \hat{\bar{S}}_{i j} \hat{\bar{S}}_{i j}\right\rangle^{-1},
\end{aligned}
$$

where the top hat "^^" represents test filtering. The angle brackets " \langle\rangle " denote global averaging (averaging over the whole computational domain). For unstructured grids, it is difficult to define the test filtering width $\hat{\bar{\Delta}}_{m}$ since the shape of the neighboring elements might be different from each other. In the present work, we use the cubic root of the summation of the neighboring elements' volumes as the test filtering width. The test filtering of a physical variable is computed using the simple averaging of the variables defined at neighboring elements, i.e.,

$$
\begin{gathered}
\hat{\bar{\phi}}=\frac{1}{F+1}\left(\bar{\phi}_{c_{0}}+\sum_{f=1}^{F} \bar{\phi}_{c_{f}}\right), \\
\hat{\bar{\Delta}}_{m}=\left(\Delta V_{c_{0}}+\sum_{f=1}^{F} \Delta V_{c_{f}}\right)^{1 / 3},
\end{gathered}
$$

where $\hat{\bar{\phi}}$ is the test-filtered variable, $F$ is the number of neighboring cells of a given cell $c_{0}$. In the dynamic procedure, Eq. (5) is firstly employed to get the coefficient $C$ dynamically. Then it is substituted into Eq. (4) to calculate the turbulence viscosity coefficient $v_{\mathrm{t}}$. Small subgrid-dissipation in the laminar and transition flow regions is guaranteed by $\bar{B}_{\beta}$, which could vanish in those regions.

It can be seen in Fig. 1 that the eddy-viscosity coefficient is positive and almost zero near the contour of the spanwise vorticity. Therefore, The Vreman SGS model in company with a dynamic scheme could generate vanishing dissipation for laminar flows and transition regimes, without any free parameters. This advantage is particularly suitable to flows around a cylinder at subcritical Reynolds numbers. Meanwhile, the artificial clip technique is no longer needed to guarantee the positive eddy-viscosity coefficients.

\subsection{Discretization of the Navier-Stokes equations}

The fractional step method [15] is used to decouple the pressure term from the momentum equations. This computational procedure can be formulated as

$$
\begin{gathered}
\frac{\bar{u}_{i}^{*}-\bar{u}_{i}^{n}}{\Delta t}+\left(\frac{3}{2} \frac{\partial \bar{u}_{i}^{n} \bar{u}_{j}^{n}}{\partial x_{j}}-\frac{1}{2} \frac{\partial \bar{u}_{i}^{n-1} \bar{u}_{j}^{n-1}}{\partial x_{j}}\right) \\
=\frac{1}{\operatorname{Re}} \frac{\partial^{2}}{\partial x_{j} \partial x_{j}}\left(\frac{\bar{u}_{i}^{*}+\bar{u}_{i}^{n}}{2}\right)-\frac{\partial \bar{\tau}_{i j}^{n}}{\partial x_{j}}
\end{gathered}
$$
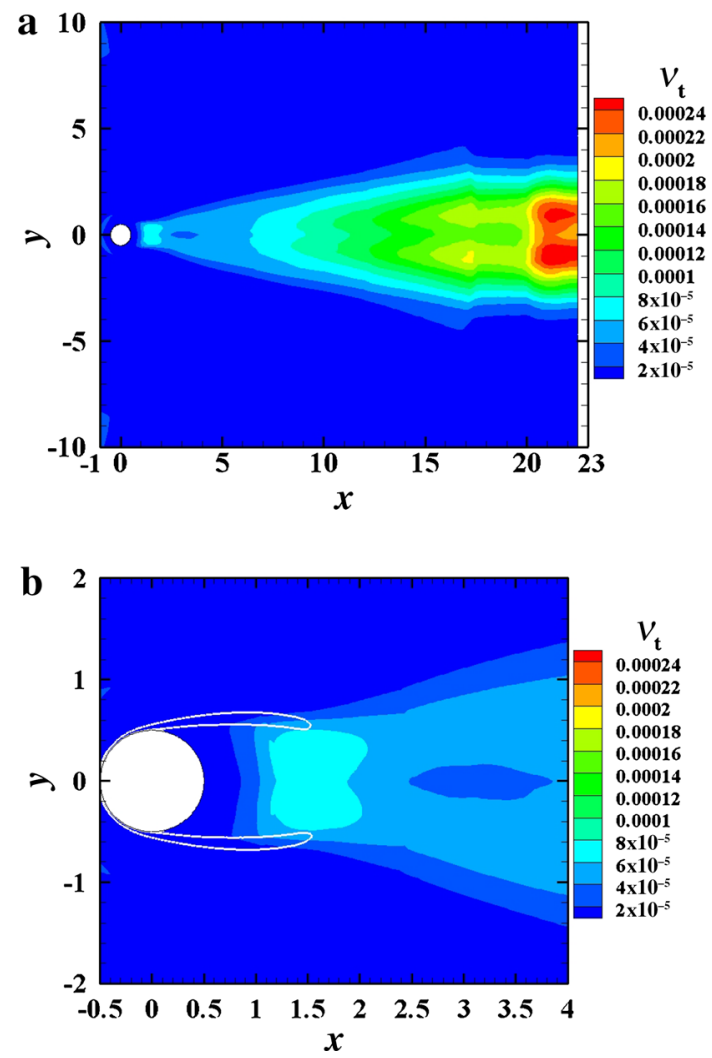

Fig. 1 a Mean eddy-viscosity contour in LES of flows passing a circular cylinder at Reynolds number 3900. b The zoom-in portion near the cylinder. The white lines are the contours of mean spanwise vorticity of the value \pm 5 .

$\bar{u}_{i}^{n+1}=\bar{u}_{i}^{*}-\Delta t \frac{\partial \bar{p}^{n+1}}{\partial x_{i}}$.

To advance the momentum equation in time, an explicit 2 nd order Adams-Bashforth scheme is used for the convection term and a 2nd order Crank-Nicholson scheme is used for the viscous term. The intermediary velocity $\bar{u}_{i}^{*}$, resulting from a preliminary advancing step, do not satisfy the continuity equation. The correction of $\bar{u}_{i}^{*}$ based on the pressure-gradient results in the velocity field at $n+1$ step, which satisfies the continuity equation. A Poisson equation for the pressure $\bar{p}^{n+1}$ can be derived from Eq. (8) and the divergence-free condition:

$\frac{\partial^{2} \bar{p}^{n+1}}{\partial x_{i} \partial x_{i}}=\frac{1}{\Delta t} \frac{\partial \bar{u}_{i}^{*}}{\partial x_{i}}$.

The finite volume method is used to discretize the space derivatives. The velocity vector and pressure are defined at cell centers. The pressure gradient correction in the correction step could introduce artificial pressure work in the calculation [11]. It should be noted that Ham et al. [11] introduced a discretization of the Laplacian operator for the pressure equation that can reduce the artificial work. 


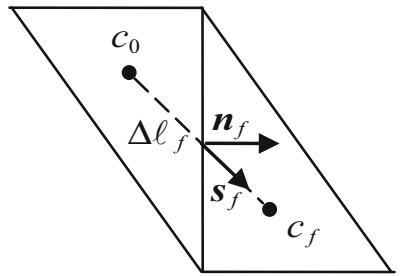

Fig. 2 Two neighboring cells $\left(c_{0}\right.$ and $\left.c_{f}\right)$ in an unstructured grid. The surface between the two cells (on which the velocity flux $U_{f}^{n}$ is defined) has an area of $A_{f} . \Delta \ell_{f}$ is the distance between the two cells. The velocity $\bar{u}_{i, c_{0}}^{n}$ and pressure $\bar{p}_{c_{0}}^{n}$ are defined at cell centers

Figure 2 shows two neighboring cells $\left(c_{0}\right.$ and $\left.c_{f}\right)$ in an unstructured grid. $\Delta \ell_{f}$ is the distance between the two cells. $\boldsymbol{n}_{f}$ is the norm direction of the face between $c_{0}$ and $c_{f}$. $A_{f}$ is the area of the face and $U_{f}$ is velocity flux on the face. $\boldsymbol{s}_{f}$ is the direction vector connecting two cell centers. Besides the velocity vector at the cell centers, $U_{f}$ is also treated as an independent variable. The conservation of discrete kinetic energy requires that the velocity on the face between two neighboring cells is computed by a simple average of two velocities at cell centers, i.e.,

$\frac{\partial \bar{u}_{i}^{n} \bar{u}_{j}^{n}}{\partial x_{j}} \approx \frac{1}{\Delta V_{c_{0}}} \sum_{f=1}^{F} A_{f} U_{f}^{n} \cdot \frac{\bar{u}_{i, c_{0}}^{n}+\bar{u}_{i, c_{f}}^{n}}{2}$.

At the projection step, the pressure gradient can be approximated by using the Gauss-Green theorem as

$\frac{\partial \bar{p}^{n+1}}{\partial x_{i}} \approx \frac{1}{\Delta V_{c_{0}}} \sum_{f=1}^{F} A_{f} n_{i, f} \cdot \frac{\bar{p}_{c_{0}}^{n+1}+\bar{p}_{c_{f}}^{n+1}}{2}$.

A correction term [11] is also added to the discretized Laplacian operator to minimize the unphysical work due to the numerical errors in computing pressure gradient, i.e.,

$$
\begin{aligned}
\frac{\partial^{2} \bar{p}^{n+1}}{\partial x_{i} \partial x_{i}} \approx & \frac{1}{\Delta V_{c_{0}}} \sum_{f=1}^{F} A_{f}\left\{\left(\boldsymbol{n}_{f} \cdot \boldsymbol{s}_{f}\right) \frac{\bar{p}_{c_{f}}^{n+1}-\bar{p}_{c_{0}}^{n+1}}{\Delta \ell_{f}}\right. \\
& +\frac{1}{2}\left(\frac{\partial \bar{p}_{c_{0}}^{n+1}}{\partial x_{i}}+\frac{\partial \bar{p}_{c_{f}}^{n+1}}{\partial x_{i}}\right) \\
& \left.\times\left[n_{i, f}-\left(\boldsymbol{n}_{f} \cdot \boldsymbol{s}_{f}\right) s_{i, f}\right]\right\} .
\end{aligned}
$$

The right hand side of the Poisson Eq. (9) can be discretized as

$$
\frac{1}{\Delta t} \frac{\partial \bar{u}_{i}^{*}}{\partial x_{i}} \approx \frac{1}{\Delta t} \frac{1}{\Delta V_{c_{0}}} \sum_{f=1}^{F} A_{f} U_{f}^{*},
$$

where

$U_{f}^{*}=\frac{1}{2} n_{i, f}\left(\bar{u}_{i, c_{0}}^{*}+\bar{u}_{i, c_{f}}^{*}\right)$.

Equations (12)-(14) give the discretized form of the pressure equation. The velocity field is advanced by combining Eqs. (7), (8), and (11). The continuity equation requires that the flux $U_{f}^{n+1}$ at $(n+1)$-th step should be computed as

$$
\begin{aligned}
& U_{f}^{n+1}=U_{f}^{*}-\Delta t\left\{\left(\boldsymbol{n}_{f} \cdot \boldsymbol{s}_{f}\right) \frac{\bar{p}_{c_{f}}^{n+1}-\bar{p}_{c_{0}}^{n+1}}{\Delta \ell_{f}}\right. \\
& \left.+\frac{1}{2}\left(\frac{\partial \bar{p}_{c_{0}}^{n+1}}{\partial x_{i}}+\frac{\partial \bar{p}_{c_{f}}^{n+1}}{\partial x_{i}}\right)\left[n_{i, f}-\left(\boldsymbol{n}_{f} \cdot \boldsymbol{s}_{f}\right) s_{i, f}\right]\right\} .
\end{aligned}
$$

The resulting flux $U_{f}^{n+1}$ on the surface is needed in Eq. (10) for calculating the convection term. The spatial derivatives used in the calculation of SGS stress are also obtained using a second-order finite volume discretization [16].

\subsection{Computational set-up and code parallelization}

The schematic representation of the computational domain is shown in Fig. 3. The origin of the coordinate system is defined at the center of the cylinder. The $x$-axis coincides with the oncoming flow direction. $z$-axis coincides with the spanwise direction. The $y$-axis is perpendicular to the $x-y$ plane. The $x$-coordinate of the computation domain is from -20 to 22.7. The $y$-coordinate is from -20 to 20 . The length of the cylinder in the spanwise direction is 3.2.

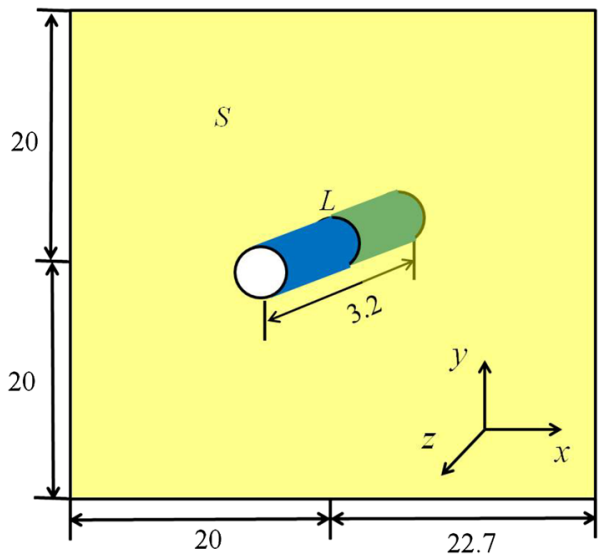

Fig. 3 Schematic representation of the computation domain. $x, y$ and $z$ are the coordinates in the streamwise, normal and spanwise directions, respectively. $S$ is the section area and $L$ is the length of intersection line in Eq. (18) 

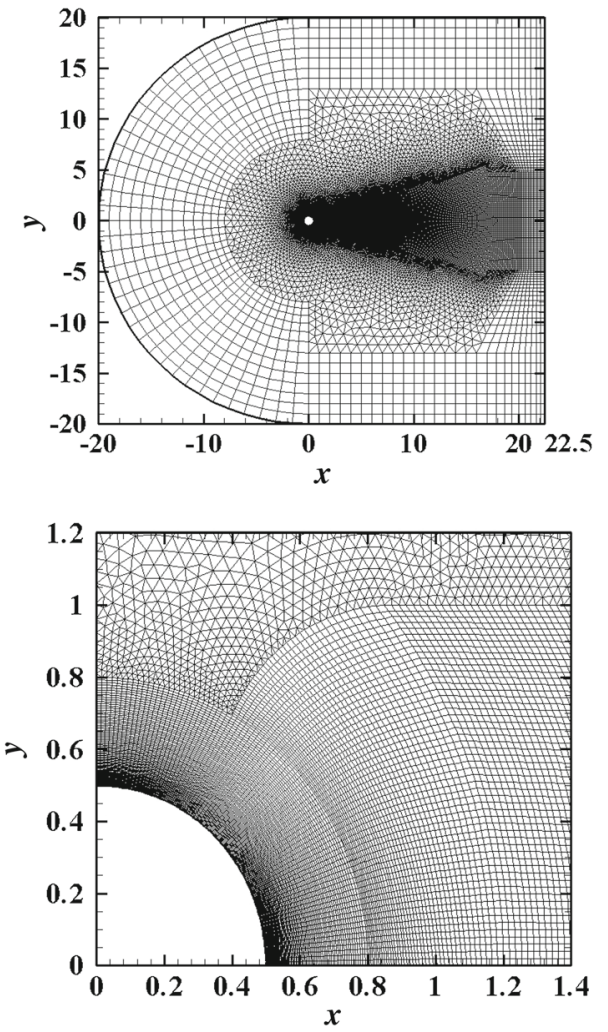

Fig. 4 Hybrid unstructured grid in cross-section and an enlarged view near the back of the cylinder. Cubic cells are used in the boundary layer, the wake and far field region. Prism cells are used to patch the far field and the wake. The cells are arranged almost perpendicular to each other to best preserve accuracy

A constant oncoming velocity is prescribed at the inlet. On the cylinder surface, a non-slip and impenetrable boundary condition is used. The top and bottom boundaries are treated as slip and impenetrable walls. At the outlet, a convective boundary condition is used. The periodic boundary condition is used in the spanwise direction. The pressure at all boundaries is zero-gradient.

Cubic and prism hybrid grids are used to discretize the computation domain. The cubic cells are used in the boundary layer and the wake region to preserve the 2nd order accuracy of the calculation. The grids are refined in the boundary layer and shear layer regions to resolve the small flow structures. In the far field region, cubic cells are used to reduce the number of cells. Prism cells are used to patch the region between the wake and the far field. There are $7.5 \times 10^{4}$ surface elements in a cross-section shown in Fig. 4. Eighty layers are used in the spanwise direction. The total number of cells is about $6 \times 10^{6}$.

$M P I$ is used to parallel the code. The computational grid is partitioned by METIS [17]. About $90 \%$ computational resources are used in solving the pressure equation. The algebraic multi-grid method (AMG) is used to solve the pressure equation. The solving procedure uses the Boomer_AMG

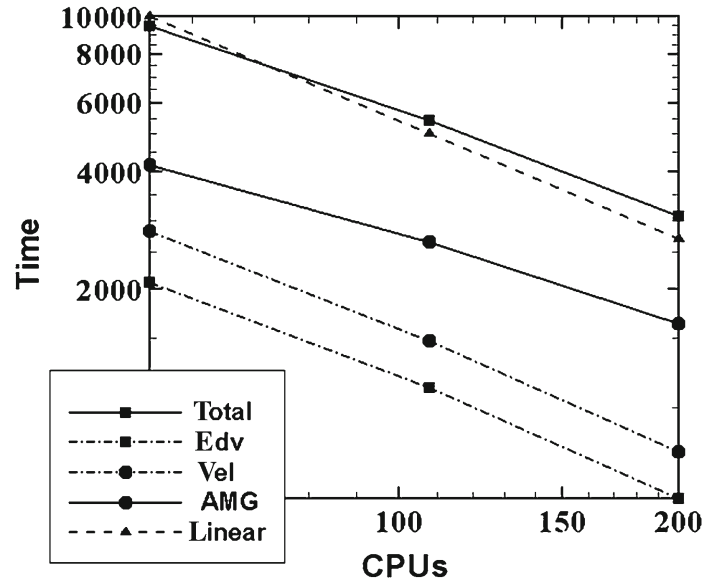

Fig. 5 The time consumed in the calculation of flow pass a cylinder by using 54, 108 and 200 CPUs. Dash line, with triangles on it, is the linear acceleration rate. "Total" denotes the total time used in one calculation. "AMG" denotes the time used to solve the pressure equation. The speedup in the parallelization is close to linear

solver of HYPRE package [18]. As shown in Fig. 5, a linear speed-up has been achieved in the parallel computing test.

\subsection{Code validation: laminar flow around a circular cylinder at $\mathbf{R e}=\mathbf{2 7 0}$}

For validation purposes, the laminar flow around a circular cylinder at $R e=270$ is first simulated using the code. Different from the $R e=3900$ case, the length of the cylinder in the spanwise direction is 10.0 to fully contain the large spanwise structures. The SGS model is not used in the laminar calculation. Figure 6 shows the vortex structures, for the present results and the experimental results of Williamson [9]. The part above the dashed line is extended according to the periodic boundary condition to facilitate the comparison with the experimental result below. Large and small streamwise vortex structures are clearly seen (such wake modes are termed as "B-instability" modes). The wave length in this wake mode is about 1.0D. The distance between two neighboring spanwise vortices is about $5 \mathrm{D}$. The vortex structures shown in the two figures are very similar.

\section{Curle's integral of Lighthill's acoustic analogy}

Lighthill's acoustic analogy [19] is used to obtain the sound field from the turbulent source calculated by LES. Curle's integral [20] is used to solve the Lighthill equation. The integral takes account of the influence of the solid wall of the cylinder but neglects the influence of sound on the flow field. Curle's solution can be written in a simplified form, if the observation point is far from the source region and if the 


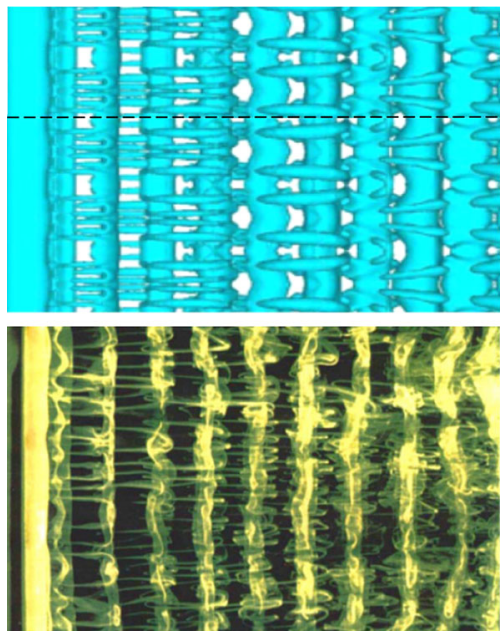

Fig. 6 Iso-vorticity surfaces from the present calculation $(u p)$ and the flow visualization in the experiment (down)

compact source assumption holds:

$$
\begin{gathered}
p\left(\boldsymbol{x}^{\prime}, t\right)-p_{\infty} \approx \frac{M_{\infty}^{2}}{4 \pi} \frac{\partial^{2}}{\partial t^{2}} \int_{V} \frac{\left(x_{i}^{\prime}-x_{i}\right)\left(x_{j}^{\prime}-x_{j}\right)}{\left|\boldsymbol{x}^{\prime}-\boldsymbol{x}\right|^{3}} \\
\times \bar{T}_{i j}\left(\boldsymbol{x}, t-\left|\boldsymbol{x}^{\prime}-\boldsymbol{x}\right| M_{\infty}\right) \mathrm{d} V-\frac{M_{\infty}}{4 \pi} \frac{\partial}{\partial t} \\
\times \oint_{S} \frac{x_{i}^{\prime}-x_{i}}{\left|\boldsymbol{x}^{\prime}-\boldsymbol{x}\right|^{2}} \bar{p}\left(\boldsymbol{x}, t-\left|\boldsymbol{x}^{\prime}-\boldsymbol{x}\right| M_{\infty}\right) n_{i} \mathrm{~d} S,
\end{gathered}
$$

where $\boldsymbol{x}^{\prime}$ is the position of the observation point. The volume of the source region is $V$. Solid surface is denoted as $S$, the speed of sound denoted as $a$. The Mach number is defined as $M_{\infty}=U_{\infty} / a$. The Lighthill stress tensor $\bar{T}_{i j}$ is defined as

$\bar{T}_{i j}=\boldsymbol{u}_{i} \boldsymbol{u}_{j}$

For a cylinder with an infinitely long span, the source region is compact in the streamwise $\left(x_{1}\right)$ and crosswise $\left(x_{2}\right)$ directions. In the spanwise direction $\left(x_{3}\right)$, it is non-compact. Using the compact source assumption in $x_{1}$ and $x_{2}$ direction, the integral in Eq. (16) can be further simplified as

$$
\begin{aligned}
& p\left(\boldsymbol{x}^{\prime}, t\right)-p_{\infty} \approx \frac{M_{\infty}^{2}}{4 \pi} \int_{-\infty}^{\infty}\left[\frac{\left(x_{i}^{\prime}-\delta_{i 3} x_{i}\right)\left(x_{j}^{\prime}-\delta_{j 3} x_{j}\right)}{d^{3}}\right. \\
& \left.\times \frac{\partial^{2}}{\partial t^{2}} \int_{S^{\prime}\left(x_{3}\right)} \bar{T}_{i j}\left(\boldsymbol{x}, t-\mathrm{d} M_{\infty}\right) \mathrm{d} S^{\prime}\left(x_{3}\right)\right] \mathrm{d} x_{3} \\
& \quad-\frac{M_{\infty}}{4 \pi} \int_{-\infty}^{\infty}\left[\frac{x_{i}^{\prime}-\delta_{i 3} x_{i}}{d^{2}}\right. \\
& \left.\quad \times \frac{\partial}{\partial t} \oint_{L\left(x_{3}\right)} \bar{p}\left(\boldsymbol{x}, t-d M_{\infty}\right) n_{i} \mathrm{~d} L\left(x_{3}\right)\right] \mathrm{d} x_{3},
\end{aligned}
$$

where $\delta$ is the Kronecker symbol. The distance between the observation point $\left(x_{1}, x_{2}, x_{3}\right)$ and a source region point $\left(x_{1}^{\prime}, x_{2}^{\prime}, x_{3}^{\prime}\right)$ is defined as $d=\left[x_{1}^{\prime 2}+x_{2}^{\prime 2}+\left(x_{3}^{\prime}-x_{3}\right)^{2}\right]^{1 / 2}$. The area of an integral section $S^{\prime}\left(x_{3}\right)$ is perpendicular to $x_{3}$. The arc length of the integral is denoted as $L\left(x_{3}\right)$ (as shown in Fig. 3).

\section{Results and discussion}

\subsection{Turbulent flow around a circular cylinder at $\boldsymbol{R e}=\mathbf{3 9 0 0}$}

The flow at $R e=3900$ is in the sub-critical regime. The spanwise length of the cylinder is 3.2 in this case. According to Kravchenko et al. [21], this length is sufficiently large for the streamwise vortex structures to evolve. Figure 7 shows the mean streamwise velocity $U_{x}$ at $y=0$, where $x=0.5-$ 4.5 is the region of vortex formation. This region can be further separated into the backflow zone $(x \approx 0.5-1.5)$ and the recovering zone $(x \approx 1.5-4.5)$. The region at $x=4.5-$ 10 is the one near wake. The mean streamwise velocity is compared with the previous results from computations and experiments. The solid line is from Kravchenko et al. [21]. Theyscheme based on B-spline. The dash-dotted line is from Beaudan et al. [22]. They used a 5th order upwind biased finite difference scheme. The SGS model used in these two simulations was the dynamic Smagorinsky model. The experimental data for the vortex-formation region is from the particle image velocimetry (PIV) result of Lourenco et al. [23]. The near wake result is from Ong and Wallace [24] in which the hot-wire velocimetry was used. The present results are in consistence with the experimental ones. Overall, it is comparably good with the one from the up-wind scheme but

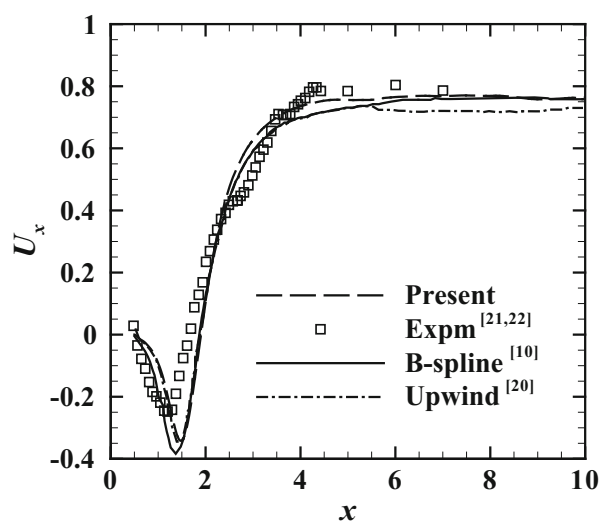

Fig. 7 Ensemble averaged streamwise velocities at $y=0$. The solid line is the computation result from Kravchenko et al. [21] using the B-spline method. The dash-dotted line is the numerical result from Beaudan [22] using an upwind scheme. The dashed line is the result from the present work. The squares are experimental results. The near wake parts are from Lourenco et al. [23] while the last four points in the far wake are from Ong et al. [24] 
worse than the one from the B-spline scheme. The reason is that we use a second order finite difference scheme while the $\mathrm{B}$-spline scheme is of higher-order. All of the results from three numerical simulations predict the trends of the experimental ones with some discrepancies. The B-spline presents the best one for $x<4$, but the present results looks best near $x=4$. The results from the present simulation and B-spline looks very similar for $x>6$ while the up-wind gives the worst results.

The profiles of mean streamwise velocity $\left(U_{x}-y\right)$ at $x=$ $1.06,1.54,2.02$ are compared with several computational and experimental results in Fig. 8. The trend of the mean velocity profile in the present work is consistant with that of the experiment. The present result (dashed line) is very close to the computational result in Ref. [22] (dash-dotted line). In Fig. 9, the profiles of mean crosswise velocity $\left(U_{y}-\right.$ $y)$ are also compared with the results in [21]. It is difficult to measure the crosswise velocity in the experiments (the relative error could exceed $50 \%$ [22]). Thus the experimental data are not included in this comparison. There are some discrepancies between the two computational results, but the differences are small and within the $5 \%$ of the magnitude. The profile in Fig. 9a is located at $x=1.06$, which is in the separation bubble region. The four points of local minimal (or maximal) values of mean streamwise velocity arise from the swirling flow in the separation bubble behind the cylinder. The profiles in Fig. 9b, c are located outside the separation bubble. As a result, only two points of local minimal (or maximal) values are observed.

The flow in the near wake region is fully developed turbulence. Figure 10 shows the profiles of one component of the mean Reynolds stress tensor $R_{11}=\left\langle u_{1}^{\prime} u_{1}^{\prime}\right\rangle$ at $x=6,7,10$. All computational results exhibit the same trend. The present work (dash line) is closer to the B-spline results (solid line) [21], and larger than the up-wind scheme results (dash-dotted line) [22]. The Reynolds stress component $R_{11}$ declines gradually with increasing $\mathrm{x}$. This trend is also the same as that in the experiment [24].

Figure 11 shows the contours of mean spanwsie vorticity in the $x-y$ plane. The contours are averaged in the spanwise direction $(z)$ and in time. Two free shear layers are separated from the two boundary layers of the cylinder. The mean vorticity magnitude in the shear layer gradually reduces to zero with increasing $x$. The length of the shear layer (defined by using a shreshold vorticity manitude of 10.0 ) is about 1.5 , which is about the length of the separation bubble. The drastic reduction of spanwise vorticity in the near wake region might be caused by the prevailing streamwise vortex structures, which are rolled up by the shear-layer instabillity in the spanwise. This phenomenon could be seen more clearly in Fig. 12, where the vortex structures are represented by the $Q$ criterion.
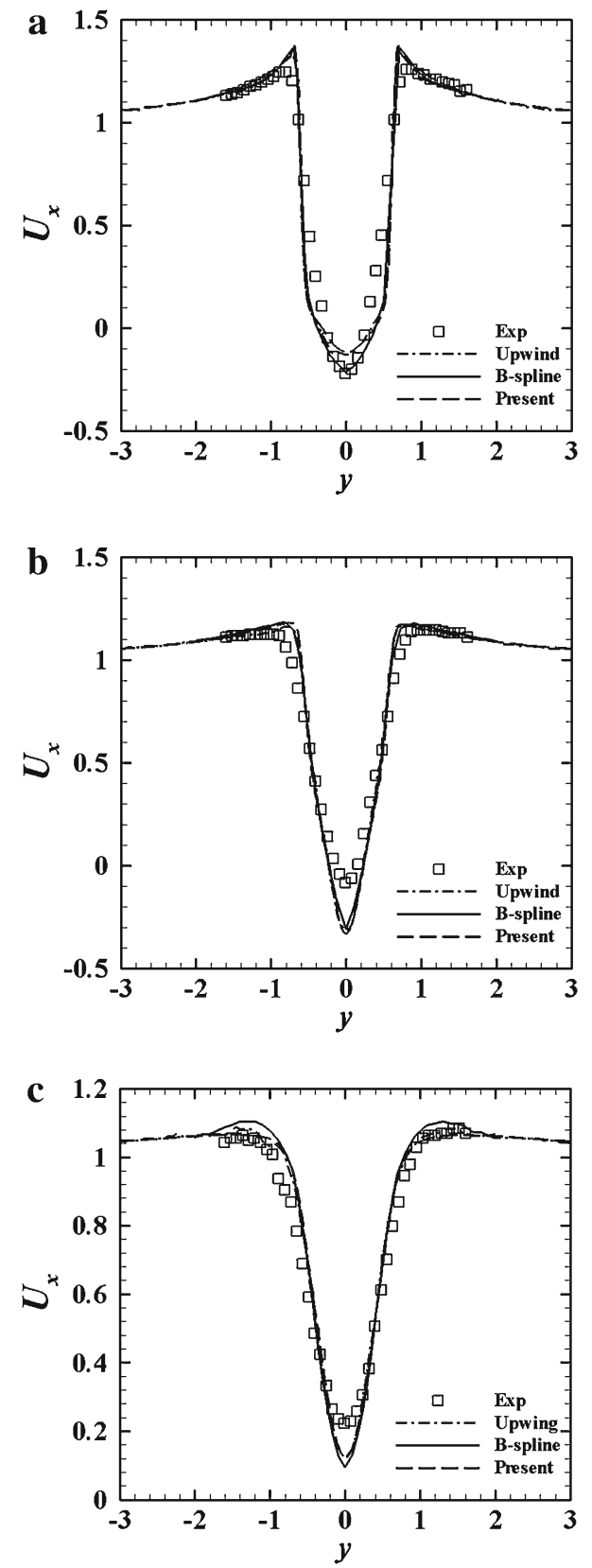

Fig. 8 The ensemble averaged streamwise velocities at different streamwise positions. The meanings of symbols are the same as Fig. 7. $\mathbf{a} x=1.06$. b $x=1.54$. $\mathbf{c} x=2.02$

The vortex structures in the flow field are also visualized using the $Q$ criterion. $Q$ is defined as the second invariant of the velocity gradient tensor:

$Q=-\frac{1}{2} \frac{\partial \boldsymbol{u}_{i}}{\partial x_{j}} \frac{\partial \boldsymbol{u}_{j}}{\partial x_{i}}$

Figure 12 shows the iso-surface of $Q=0$, together with the three-dimensional streamlines and the pressure contours on the plane of $z=0$. The iso-surface and streamlines are dyed 

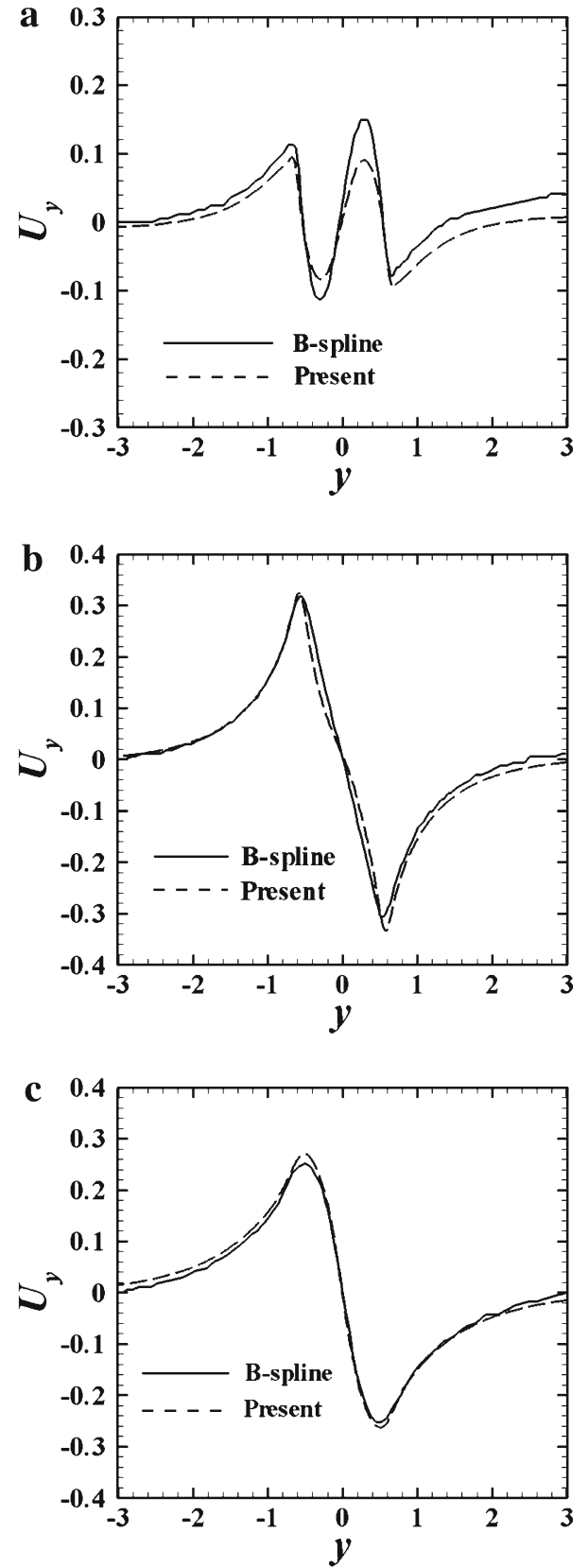

Fig. 9 The ensemble averaged transverse velocities at different streamwise positions. The solid line is the result in Ref. [21]. The dash line is the results from the present work. a $x=1.06$. b $x=1.54$. $\mathbf{c} x=2.02$

using the magnitude of $x$ and $z$ coordinate respectively. In the turbulent wake, both small turbulent structures and large structures (the vortex street) are clearly seen.

\subsection{Sound radiation}

The sound pressure fluctuation $p^{\prime}\left(\boldsymbol{x}^{\prime}, t\right)$ in the present paper is defined as

$p^{\prime}\left(\boldsymbol{x}^{\prime}, t\right)=p\left(\boldsymbol{x}^{\prime}, t\right)-p_{\infty}$ $\mathbf{a}$
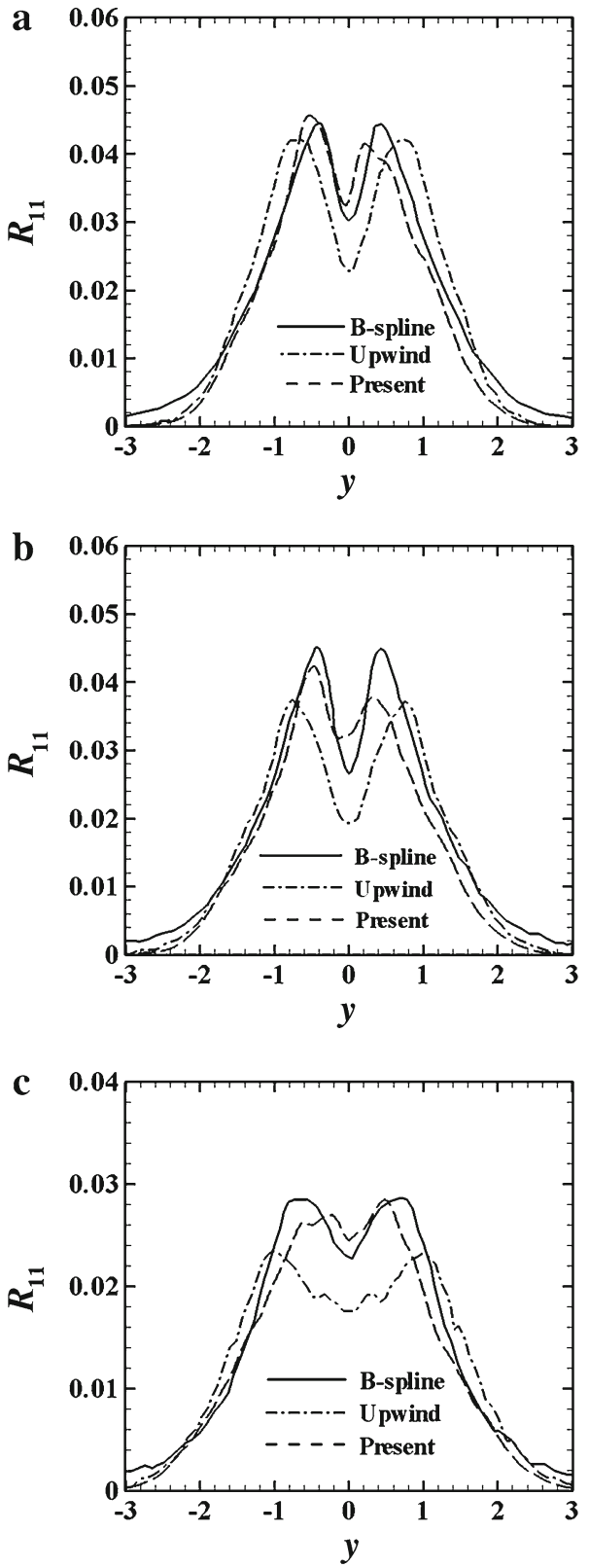

Fig. 10 The ensemble averaged streamwise Reynolds stresses at different streamwise positions. The solid line is the results in Ref. [21]. The dash-dotted line is the results in Ref. [22]. The dashed line is results from the present work. a $x=6$. b $x=7$. $\mathbf{c} x=10$

To be compatible with the assumption that the flow in the source region is governed by the incompressible NavierStoke equations, the Mach number $M_{\infty}$ is set to 0.1 in computing the sound radiation. The contours of sound pressure $p^{\prime}$ are shown in Fig. 13a. This sound pressure is the summation of the sound pressure fluctuation on the cylinder surface ( $p_{S^{\prime}}^{\prime}$ the second part of the right hand side in Eq. (18)) and the sound pressure fluctuation from the wake of the cylinder ( $p_{L}^{\prime}$ the first part of the right hand side in Eq. (18)). Figure $13 \mathrm{~b}$ shows the distribution of $p_{S^{\prime}}^{\prime}$ (the volume integral 


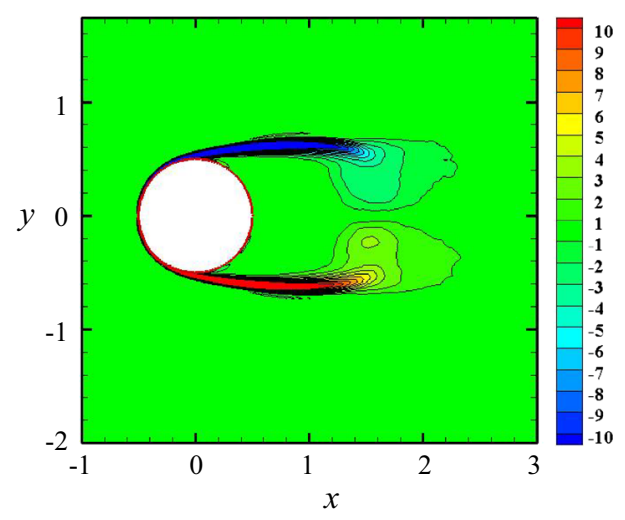

Fig. 11 Ensemble averaged spanwise vorticity contours in the vortex formation region

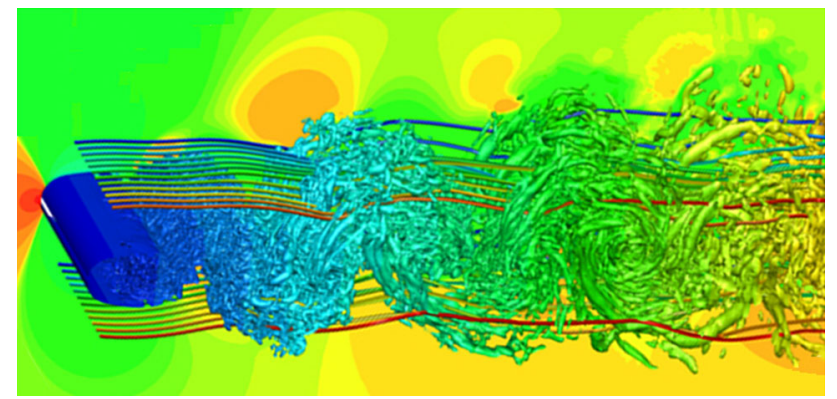

Fig. 12 Iso-surface of $Q=0$ for the instantaneous flow field together with three-dimensional streamlines and pressure contours

in Eq. (9)). It exhibits the characteristics of a quadrupole. Figure $13 \mathrm{c}$ shows the distribution of $p_{L}^{\prime}$ (the surface integral in Eq. (9)). It exhibits the characteristics of a dipole. As shown in this figure, the total sound pressure behaves more like that of a dipole. That is because $p_{L}^{\prime}$ decays as $d^{-1}$, while $p_{S^{\prime}}^{\prime}$ decays as $d^{-2}$ according to Eq. (9). Thus $p_{L}^{\prime}$ is dominating in the far field.

We also consider the sound pressure at two observation points (Fig. 14c), which are the same distance away from the center of the cylinder, namely, point $A$ at $(-1,125)$ (right above the cylinder) and point $B$ at $(-125,0)$ (in front of the cylinder). As the cylinder is supposed to be infinitely long in computing the sound radiation, the sound pressure should be independent on the spanwise location where the sound radiation is computed. In the present work, we choose to compute the sound radiation at the center plane $(z=1.6)$. The flow field information in the source region is repeated ten times periodically along the spanwise direction, which is long enough to be almost unchanged as the cylinder becomes longer. Figure 14 shows the evolution of sound pressure fluctuation with time. The magnitude of sound pressure fluctuation at point $\mathrm{A}$ is much larger than that at point $\mathrm{B}$. This means that the sound pressure magnitude has directivity. The dominating frequency at point $\mathrm{A}$ is the same as the frequency of lift force. The dominating frequency at point $\mathrm{B}$ is the same
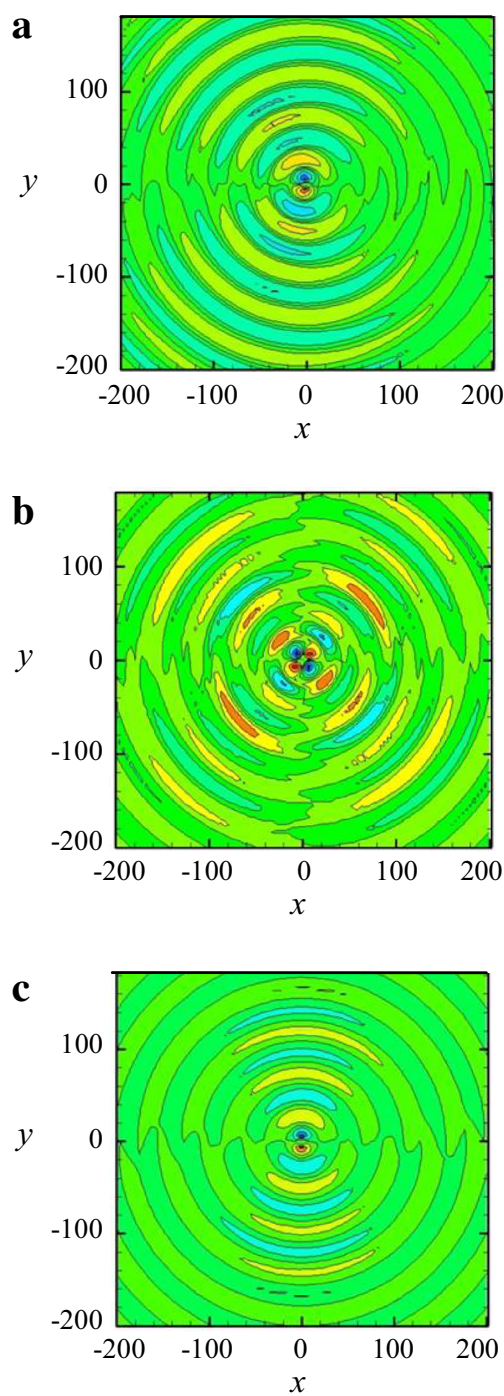

Fig. 13 Sound pressure and its two parts in Curle's acoustic analogy. Total sound pressure $p^{\prime}$ in a. Quadrupole part $p_{s}^{\prime}$ in b. Dipole part $p_{L}^{\prime}$ in $\mathbf{c}$

as the frequency of drag force. This could be seen from the Curle's integral. The dominating dipole sound is from the surface integral in Eq. (18). Only the first component of the normal direction $\left(n_{1}\right)$ contributes to the surface integral, if the observation point is in front of the cylinder. The effects of other the two components are excluded due to the dot-production of the surface normal direction and the observation direction (from center of the cylinder to the front of it). The surface integral with the first component $\left(n_{1}\right)$ of surface normal direction is drag force, when the Reynolds number is high and the viscous force could be neglected compared to the force caused by pressure difference. The same is true in the normal direction. Therefore, the sound signal at point $\mathrm{B}$ is related to drag force and the one at point A related to lift force. 

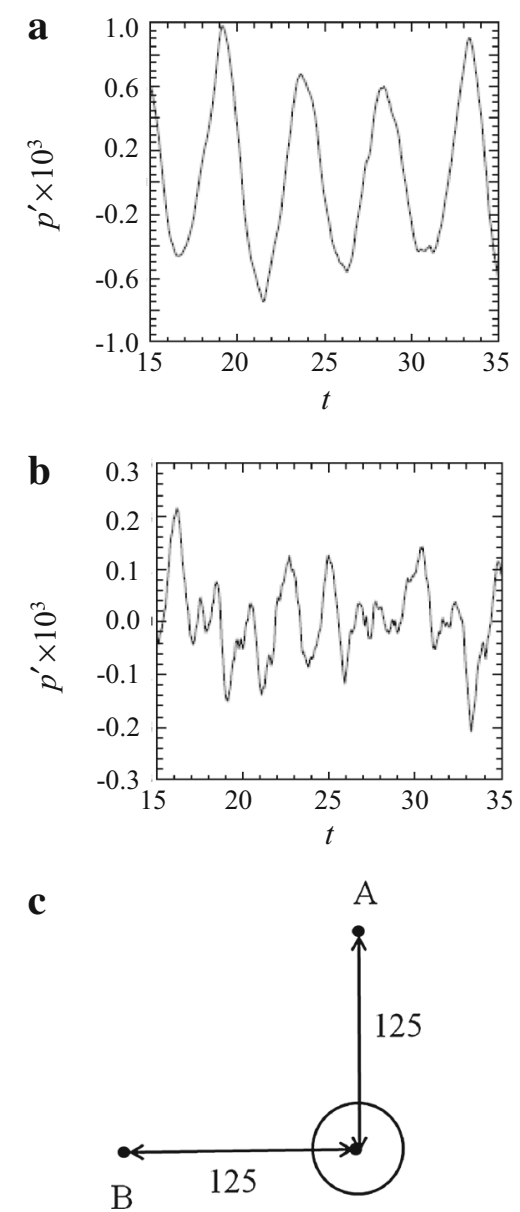

Fig. 14 Sound pressures at observation points $A(0,125)$ and $B$ $(-125,0)$. a Time serial of sound pressure fluctuation at point A. b Time serial of sound pressure fluctuation at point B. c Schematic representation of observation points $A$ and $B$. a Observation point $A(0,125)$. b Observation point $\mathrm{B}(-125,0)$. c Observation points A and B

The vortex shedding frequency of the Karman vortex street $\left(f_{0}=0.20\right)$ determines the frequency of the far field sound at point A. In the experimental study of Cardell [25], the shedding frequency of the Karman vortex street is about $0.215 \pm 0.005$. Figure 15 shows the frequency spectra of sound radiation at points $\mathrm{A}$ and $\mathrm{B}$. The frequency is normalized by $f_{0}$. The spectra are broadband and obey the $-5 / 3$ power law in the high frequency region [26]. In the low frequency range, the peak of the spectrum for point $\mathrm{A}$ is more obvious than that of the spectrum for point $\mathrm{B}$. The dominating frequency for point $\mathrm{B}$ is twice of that for point $\mathrm{A}$. The sound pressure fluctuation spectrum at point $\mathrm{A}$ is caused by lift force, while the spectrum at point B is caused by drag force. The frequency in drag force is twice of that in lift force. This observation is in agreement with the well-known results [26]: the lift force would complete a period during the shedding of a pair of vortexes while the drag force only takes a period of a single vortex shedding from the cylinder. It is concluded that the dominant frequency is generated by the
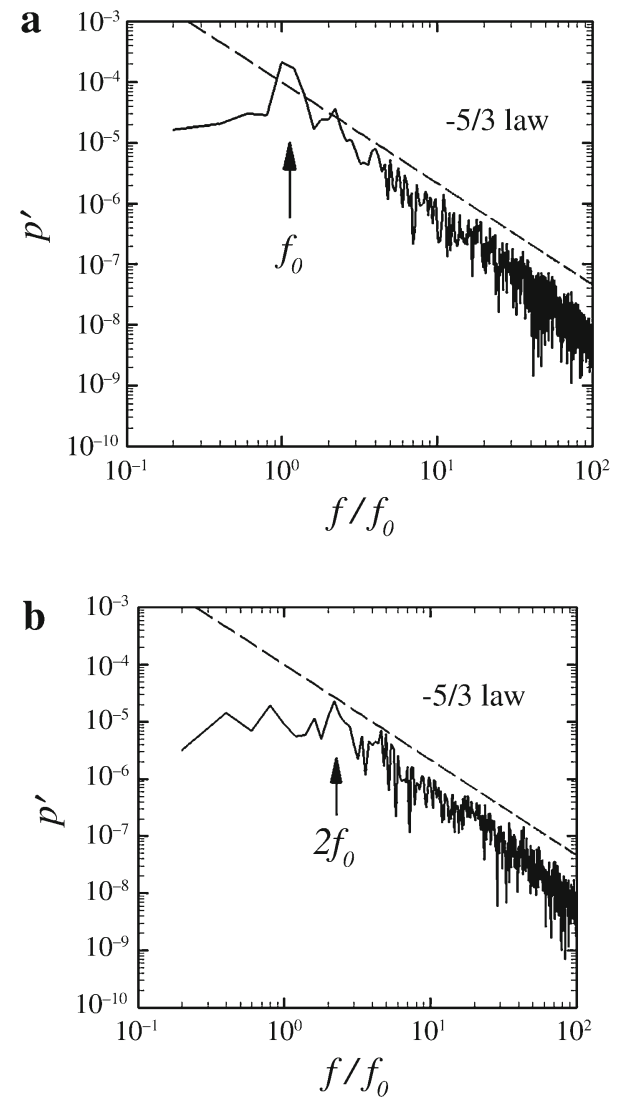

Fig. 15 Sound pressure frequency spectra at observation points A $(0,125)$ and B $(-125,0)$. a Spectrum at point A. b Spectrum at point A. The dominating frequency in $\mathbf{a}$ is half of that in $\mathbf{b}$. a Observation point $(0,125)$. b Observation point $(-125,0)$

vortex shedding process in the laminar and transition region and the $-5 / 3$ power law generated from the turbulent wakes.

\section{Summary}

The far-field noise radiated from the flows around a circular cylinder at subcritical Reynolds numbers is simulated using LES with the dynamic Vreman SGS model. The mean streamwise velocity profile of the flow field is in agreement with the experiment results. The flow in the wake generates quadrupole sound and the surface pressure fluctuations generate dipole sound. The dipole part dominates sound radiation at the far field. The sound power spectra at two observation points, right above and in front of the cylinder, are calculated. The sound spectra are broadband with a peak associated with the vortex shedding frequency. The spectra at the two points obey the $-5 / 3$ power law [26]. The dominating frequency at the front of the cylinder is twice of that at the top of the cylinder. The results show that the dynamic Vreman SGS model is suitable for the LES of far-field noise radiated from the flows around a cylinder at a subcritical Reynolds number. 
Acknowledgments The authors would like to sincerely thank Prof. D You at Pohang University of Science and Technology for his discussion and advice for the present work. This work was supported by the National Natural Science Foundation of China (Grant 11232011).

\section{References}

1. Howe, M.: Theory of vortex sound. Cambridge University Press, Cambridge (2003)

2. Marsden, A., Wang, M., Dennis, J., et al.: Trailing-edge noise reduction using derivative-free optimization and large-eddy simulation. J. Fluid Mech. 572, 13-36 (2007)

3. Yang, Q., Wang, M.: Computational study of roughness-induced boundary-layer noise. AIAA J. 47, 2417-2429 (2009)

4. Spalarta, P., Shurb, M., Streletsb, M., et al.: Towards noise prediction for rudimentary landing gear. Proc. Eng. 6, 283-292 (2010)

5. Boudet, J., Casalino, D., Jacob, M. C.: Prediction of sound radiated by a rod using large eddy simulation. AIAA Paper 2003-3217 (2003)

6. Seo, J.H., Chang, K.W., Moon, Y.J.: Aerodynamic noise prediction for long-span bodies. AIAA Paper 2006-2573 (2006)

7. Orselli, R.M., Meneghini, J.R., Saltara, F.: Two and threedimensional simulation of sound generated by flow around a circular cylinder. AIAA Paper 2009-3270 (2009)

8. Li, D., Guo, L., Zhang, X., et al.: A numerical study of a turbulent mixing layer and its generated noise. Sci. China Phys. Mech. Astron. 56, 1157-1164 (2013)

9. Williamson, C.H.K.: Vortex dynamics in the cylinder wake. Annu. Rev. Fluid Mech. 28, 477-539 (1996)

10. Murayama, M., Yokokawa, Y., Kato, H.: Computational and experimental study on noise generation from tire-axle regions of a two-wheel main landing gear. AIAA Paper 2011-2821 (2011)

11. Ham, F., You, D., Moin, P.: Discrete conservation principles in large-eddy simulation with application to separation control over an airfoil. Phys. Fluids 20, 101515 (2008)

12. Smagorinsky, J.: General Circulation Experiments with the Primitive Equations. Mon. Weather Rev. 91, 99-164 (1963)

13. Vreman, A.: An eddy-viscosity subgrid-scale model for turbulent shear flow: Algebraic theory and applications. Phys. Fluids 16, 3670 (2004)
14. You, D., Moin, P.: A dynamic global-coefficient subgrid-scale eddy-viscosity model for large-eddy simulation in complex geometries. Phys. Fluids 19, 169-182 (2007)

15. Perot, J.B.: An analysis of the fractional step method. J. Comput. Phys. 108, 51-58 (1993)

16. Mahesh, K., Constantinescu, G., Moin, P.: A numerical method for large-eddy simulation in complex geometries. J. Comput. Phys. 197, 215-240 (2004)

17. Karypis, G., Kumar, V.: A fast and highly quality multilevel scheme for partitioning irregular graphs. SIAM J. Sci. Comput. 20, 359 392 (1999)

18. Henson, V., Yang, U.: BoomerAMG: A parallel algebraic multigrid solver and preconditioner. Appl. Numer. Math. 41, 155-177 (2002)

19. Lighthill, M.J.: On sound generated aerodynamically. I. General theory. Proc. R. Soc. A 211, 564-587 (1952)

20. Curle, N.: The influence of solid boundaries upon aerodynamic sound. Proc. R. Soc. A 231, 505-510 (1955)

21. Kravchenko, A.G., Moin, P.: Numerical studies of flow over a circular cylinder at $\operatorname{ReD}=3900$. J. Comput. Phys. 197, 215-240 (2004)

22. Beaudan, P., Moin, P.: Numerical experiments on the flow past a circular cylinders at sub-critical Reynolds numbers. Report No. TF62, Department of Mechanical Engineering, Stanford University, Stanford (1994)

23. Lourenco, L., Shih, C., Krothapalli, A.: Observations on the near wake of a yawed circular cylinder. Laser anemometry in fluid mechanics V. Springer-Verlog, Berlin (1993)

24. Ong, L., Wallace, J.: The velocity field of the turbulent very near wake of a circular cylinder. Exp. Fluids 20, 441-453 (1996)

25. Cardell, G.S.: Flow past a circular cylinder with a permeable wake splitter plate. [Ph.D. Thesis]. California Institute of Technology, Pasadena (1993)

26. Tang, K.F.: Numerical simulation of flow-induced noise by means of the hybrid method with LES and aeroacoustic analogy. [Ph.D. thesis]. University of Siegen, Siegen (2004) 